\title{
A fast Otsu thresholding method based on an improved 2D histogram
}

\author{
Yanli Tan ${ }^{1 *}$, Yongqiang Zhao ${ }^{2}$ \\ ${ }^{1}$ Department of Electronic Engineering, Taiyuan Institute of Technology, \\ Taiyuan, 030008 \\ China \\ ${ }^{2}$ Science and Technology Office, Taiyuan Institute of Technology, \\ Taiyuan, 030008 \\ China
}

Received: March 21, 2021. Revised: July 27, 2021. Accepted: August 9, 2021. Published: August 11, 2021.

\begin{abstract}
The regional division of a traditional 2D histogram is difficult to obtain satisfactory image segmentation results. Based on the gray level-gradient 2D histogram, we proposed a fast 2D Otsu method based on integral image. In this method, the average gray level is replaced by the gray level gradient in the neighborhood of pixels, and the edge features of the image are extracted according to the gray level difference between adjacent pixels to improve the segmentation effect. Calculating the integral image from the two-dimensional histogram reduces the computational complexity of searching the optimal threshold, thus reducing the amount of computation. The simulation results demonstrate that the proposed algorithm has better performance in image segmentation, with the increased computational speed and improved real-time capability.
\end{abstract}

Keywords-Image segmentation, integral image, 2D Otsu method, optimal threshold.

\section{INTRODUCTION}

$\mathrm{D}$ UE to simplicity and better visualization, the thresholding method plays a major role in image segmentation, an important step in image processing [1]. The Otsu method has been widely used for searching the optimal threshold, and it is also one of the popular thresholding algorithms for image segmentation [2]. The Otsu method determines the optimal threshold based on the maximum between-class variance between the object and background, but it cannot reflect the spatial relationship between pixels, leading to inaccurate segmentation of the images corrupted by noises. To obtain a better image segmentation result, Liu and Li constructed a 2D histogram by using the gray level of each pixel in an image and the average gray level of its neighborhood, and used a thresholding algorithm to partition the $2 \mathrm{D}$ histogram into four regions with two cross lines parallel to each axis, in which the threshold value was determined based on two regions along the diagonal [3]. However, there is a limitation of this method because it assumes that the probability of the pair of the gray level of a pixel near the threshold and the average gray level of its neighborhood is zero and this assumption is inconsistent with reality.

In general, the introduction of the 2D histogram inevitably increases the computational complexity and running time of an algorithm. To reduce the amount of computation, Hao and Zhu proposed a fast recursive $2 \mathrm{D}$ entropic thresholding algorithm by rewriting the formula for calculating the entropy in a recurrence form [4]. Fan and Zhao proposed a fast algorithm to convert the two-dimensional threshold to the one-dimensional threshold using a new area partition method [5]. Some scholars have studied the process of transforming images into 3D models. By using Python language program, combined with data and image processing software, the images are analyzed and processed, so that the transformed images are complete and will not be distorted [6]. Some scholars have proposed a halftone image segmentation method based on the analysis of directional 2D histogram. By reducing the number of gray levels at the initial level, the hierarchical image is segmented, and the gray levels are increased to the maximum value, so as to realize adaptive quantization of one-dimensional histogram and double nonlinear gray transformation [7]. Other scholars have studied satellite image processing, registration and pixel clipping of output satellite image sets, and realized sub-pixel offset correction of satellite images by statistical regularization and frequency domain filtering, thus realizing image enhancement processing [8]. These two methods can increase the computational speed and decrease the computational time, but they cannot meet the requirements for real-time image processing. In this work, we introduced the integral image into the 2D Otsu method and used the plus/minus operation, rather than the repeated summation commonly used to search the 
threshold in the exhaustive search; And it is proved that the introduction of the integral image can avoid the traversal over the entire graph, increase the real-time capability and reduce the amount of computation [9] [10]. Therefore, it can provide some reference for image segmentation and theoretical value for the improvement of two-dimensional histogram.

\section{IMPROVED 2D OTSU METHOD}

For a given image $f(x, y)$ with a size of $M N$ and gray level of $L$, the smoothed image $g(x, y)$ can be obtained by calculating the average gray level of the neighborhood $\left(\begin{array}{ll}k & k\end{array}\right)$ of each pixel, with the same gray level of $L$.

\section{A. Traditional 2D Histogram}

The regional division of a traditional $2 \mathrm{D}$ histogram is shown in Fig. 1. The smoothed image $g(x, y)$ was obtained by calculating the average gray level of the neighborhood of each pixel in the original image. The traditional 2D histogram is divided into four regions by two cross lines parallel to each axis. Let the $\mathrm{x}$ coordinate $f(x, y)$ represent the gray level of a given pixel, the y coordinate $g(x, y)$ represent the average gray level of pixels in its neighborhood, and $(s, t)$ represent the threshold vector.

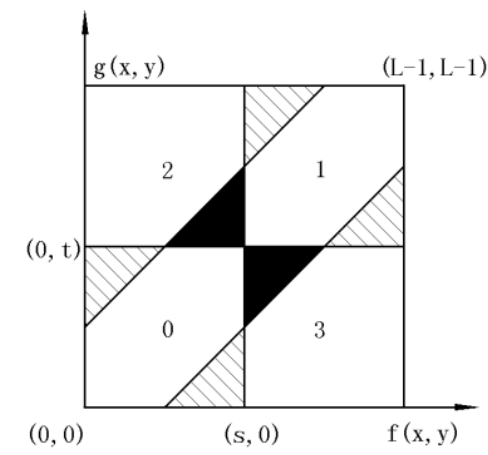

Fig. 1 regional division of a traditional $2 \mathrm{D}$ histogram

As shown in Fig. 1, region 0 and region 1 represent the object and background, respectively, because of the similarity between the gray level of the interior pixels and the average gray level of their neighborhood in these two regions. On the other hand, region 2 and region 3 represent the edge or noise because of the relatively large difference between the gray level of the interior pixels and the average gray level of their neighborhood. In the process of calculating the threshold, if the probability of occurrence of regions 0 and 1is close to 1 , the probability of all pixels in regions 3 and 4 is close to 0 . Therefore, this method has some shortcomings. For example, due to the difference between the gray level of a given pixel and the average gray level of its neighborhood, some edge pixels or noisy pixels in the slash-hatched areas in regions 0 and 1 might be incorrectly considered as interior pixels in the object or background. At the same time, this method neglects the pixels in the dark-colored areas which are near to the threshold vector and have a relatively small difference between the gray level of a given pixel and the average gray level of its neighborhood. That is to say, some pixels in the object and background are incorrectly considered as edges and noises. Therefore, this kind of regional division of the 2D histogram described above is inconsistent with reality, resulting in an unsatisfactory segmentation result.

\section{B. Improved 2D Histogram}

The improved 2D histogram uses the gray level gradient of the pixel's neighborhood to replace the average gray level. This replacement can reflect the difference in gray level between the central pixel and its neighboring pixels, and can take full use of the edge features of an image, thereby improving the segmentation effect.

In the improved 2D histogram, the y coordinate represents the absolute value of the difference between the gray level of a pixel and the average gray level of its neighborhood, $|f(x, y) \quad g(x, y)|$, rather than $\mathrm{g}(\mathrm{x}, \mathrm{y})$ used in the traditional 2D histogram. The x coordinate $f(x, y)$ represents the gray level of a pixel. For a pair $(i, j)$ with i representing the gray level of a pixel and $j$ representing the gray level gradient of its neighboring pixels, its joint probability can be calculated by (1) as follows:

$$
p_{i j}=\frac{c_{i j}}{M N}, 0 \quad i, j \quad L \quad 1
$$

where ${ }_{i=0}^{L=0} p_{i j}=1, C_{i j}$ is the number of the occurrence of the pair $(i, j)$, and it is defined as:

$$
\begin{gathered}
c_{i j}=\underbrace{M N}_{x=1 \quad M=1}(f(x, y) \quad i)(|f(x, y) \quad g(x, y)| j), \\
i=0,1, \ldots, L \quad 1, j=0,1, \ldots, L \quad 1,
\end{gathered}
$$

As shown in Fig. 2, any threshold vector $(s, t)$ can divide the 2D histogram into four regions. The gradient refers to the difference in gray levels of the pixels in the neighborhood. If the gray level of a given pixel corresponds to a lower gray level gradient, it indicates that this pixel more likely appears within the interior of the object or background, with few pixels appearing on the edge. In contrast, if the gray level of a given pixel corresponds to a higher gray level gradient of the neighborhood, it suggests that few pixels are distributed in the object and background. Therefore, in the improved 2D histogram, regions 0 and 1 correspond to the object and background, respectively, and regions 2 and 3 correspond to the edge and noise. This new approach not only ensures that regions 0 and 1 contain as many as possible pixels in the object and background, but overcomes the shortage of including noisy pixels in regions 0 and 1 in the traditional 2D histogram. 


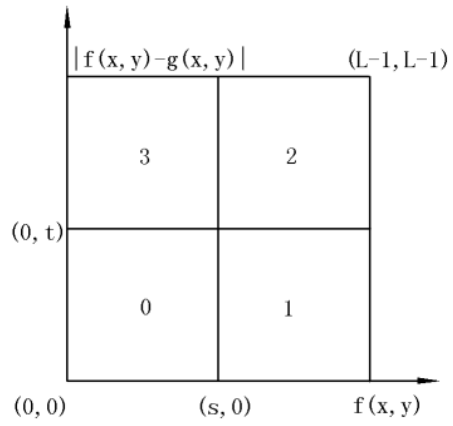

Fig. 2 regional division of the improved 2D histogram

\section{Improved 2D Otsu Method}

Two-dimensional Otsu threshold method needs to solve the optimal threshold, but at this time, it usually needs to traverse the whole solution space, so it is difficult to meet the real-time requirements of the algorithm. Therefore, the two-dimensional Otsu threshold method is improved. In the 2D histogram, two classes of pixels of $C_{0}$ and $C_{1}$ represent the object and background, respectively. The probabilities $\left(P_{0}\right.$ and $\left.P_{1}\right)$ of occurrence of these two classes at a vector $(\mathrm{s}, \mathrm{t})$ are defined by (3a) and (3b), respectively:

$$
\begin{aligned}
& P_{0}(s, t)=\underbrace{}_{\substack{i=0 \\
L_{j=0}}} p_{i j} \\
& P_{1}(s, t)={ }_{i=s+1}^{l_{j=0}} p_{i j}
\end{aligned}
$$

The average vectors of ${ }_{0}$ and ${ }_{1}$ corresponding to these two classes are defined by (4a) and (4b), respectively:

$$
\begin{aligned}
& { }_{0}(s, t)=\left({ }_{0,0}, \quad{ }_{0,1}\right)^{\mathrm{T}}=\square_{i=0}^{\square s} \square_{j=0}^{t} \frac{i p_{i j}}{P_{0}}, \square_{i=0} \square_{j=0}^{t} \frac{j p_{i j}}{P_{0}} \square \\
& { }_{1}(s, t)=\left(\begin{array}{ll}
1,0 & 1,1
\end{array}\right)^{\mathrm{T}}=\square \square_{i=s+1}^{\square} \square_{j=0}^{t} \frac{i p_{i j}}{P_{1}}, \square_{i=s+1}^{L} \square_{j=0}^{t} \frac{j p_{i j}}{P_{1}} \square
\end{aligned}
$$

The total mean vector $\left({ }_{T}\right)$ of the $2 \mathrm{D}$ histogram is defined by (5):

$$
T=\left(\begin{array}{cc}
T, 0 & T, 1
\end{array}\right)^{\mathrm{T}}=\square \square_{i=0}^{L 1} \square_{j=0} i_{i j}, \prod_{i=0}^{L 1} \square_{j=0} j p_{i j} \square
$$

If the sum of the probabilities of regions 2 and 3 is close to zero, the between-class scatter matrix $\mathrm{SB}(\mathrm{s}, \mathrm{t})$ is defined by $(6)$ :

$$
\begin{aligned}
\mathbf{S}_{B}(s, t)= & P_{0}(s, t) \square\left(\begin{array}{lll}
{ }_{0}(s, t) & T
\end{array}\right)\left(\begin{array}{lll}
{ }_{0}(s, t) & T
\end{array}\right)^{\mathrm{T}} \\
& +P_{1}(s, t) \\
{ }_{1}(s, t) & \left.{ }_{T}\right)\left(\begin{array}{ll}
{ }_{1}(s, t) & { }_{T}
\end{array}\right)^{\mathrm{T}}
\end{aligned}
$$

The trace of $\mathbf{S}_{B}$ is used to measure the between-class variance and defined by (7):

$$
\begin{aligned}
\operatorname{TrS}_{B}(s, t) & =P_{0}(s, t) \square\left(\begin{array}{cc}
0,0 & T, 0
\end{array}\right)^{2}+\left(\begin{array}{cc}
0,1 & T, 1
\end{array}\right)^{2} \square \\
& +P_{1}(s, t) \square\left(\begin{array}{cc}
1,0 & T, 0
\end{array}\right)^{2}+\left(\begin{array}{cc}
1,1 & T, 1
\end{array}\right)^{2} \square
\end{aligned}
$$

The optimal threshold $\left(s^{*}, t^{*}\right)$ for the 2D Otsu method is determined by maximizing the trace of $\mathrm{SB}(\mathrm{s}, \mathrm{t})$ :

$\left(s^{*}, t^{*}\right)=\arg \max _{0}\left\{(s, t) L 1\right.$ Tr $\left.\mathbf{S}_{B}(s, t)\right\}$

From the above calculation process, it can be seen that the method in this paper calculates the probability of vectors represented by different pixels appearing on a vector, thus reducing the dimension of solution. For the determination of the optimal threshold, it is not necessary to traverse all solution spaces, which reduces the computational complexity.

\section{INTEGRAL IMAGE}

As shown in Fig. 3(a), the gray level ii(x,y) of any point $(x, y)$ in an integral image is the sum of gray levels of all pixels in the gray area of the original image, and it is calculated by (9):

$i i(x, y)=\underbrace{}_{x x, y \text { y }} f(x, y)$

where $\mathrm{f}\left(\mathrm{x}^{\prime}, \mathrm{y}^{\prime}\right)$ is the gray level of any pixel $\left(\mathrm{x}^{\prime}, \mathrm{y}^{\prime}\right)$ in the original image. $i i(x, y)$ can be calculated by (10) and (11):

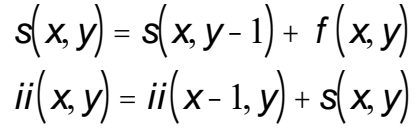

where $s(x, y)$ represents the integrals in a column under the condition of $s(x, 1)=0, i i(1, y)=0$.

An integral image can be calculated by traversing the original image once, and this method needs only a few amounts of computation. Fig. 3(b) shows the sum of gray levels in the window $\mathrm{D}$, which can be rapidly calculated using the four corresponding points, including points 1,2, 3 and 4, of the window $\mathrm{D}$, regardless of its size. That is to say, the sum of gray levels in window D can be calculated as $1+4 \quad(2+3)$. 


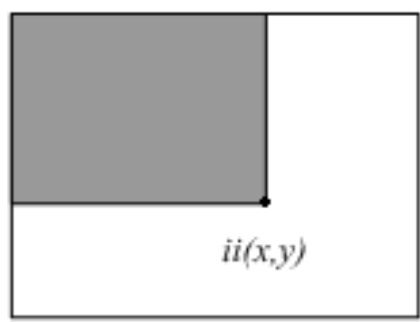

Fig. 3(a) the integral image

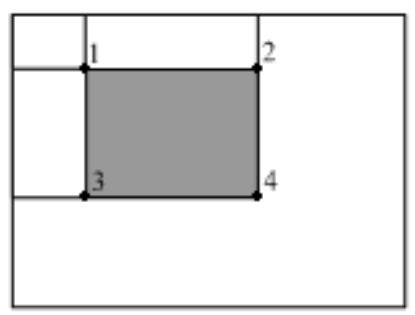

Fig. 3(b) using the integral image to calculate the sum of gray levels in window D

\section{FAST 2D OTSU METHOD}

There are a lot of summation operations for matrix areas in the $2 \mathrm{D}$ histogram. If the corresponding integral image can be first calculated from the $2 \mathrm{D}$ histogram, it will be very convenient to calculate the sum. The algorithm is described in detail below.

Firstly, the 2D histogram is calculated by traversing the original image using in (1).

Secondly, three integral images need to be calculated. As shown in (7), in order to calculate the trace of $\mathrm{SB}(\mathrm{s}, \mathrm{t})$, it needs to first calculate the probabilities $\left(P_{0}\right.$ and $\left.P_{1}\right)$ of the occurrence of two classes ( $\mathrm{C} 0$ and $\mathrm{C} 1)$ and their average vectors $\left({ }_{0}\right.$ and ${ }_{1}$ ). To do so, it needs to calculate the number of the pixels in these two classes, the sum of gray levels of the original image, and the sum of gray levels of the neighborhood. Thereby, three integral images are calculated by (12), (13) and (14), respectively.

Integral image calculated from the number of pixels:

$i i_{N}(x, y)={ }_{i=0}^{x} C_{j=0}^{y} C_{i j}$

Integral image calculated from the gray levels of the original image:

$i i_{O}(x, y)={ }_{i=0 \quad j=0}^{x} i C_{i j}$

Integral image calculated from the gray level gradient of the neighborhood: $i i_{G}(x, y)={ }_{i=0 \quad j=0}^{x} j c_{i j}$

These three integral images can be calculated by traversing the 2D histogram only once.

Finally, the optimal threshold value is searched using (7), and the fast calculation can be performed by introducing the integral image.

Let:

$$
F(s+1, t+1)=i i(s, t)+i i(L \quad 1,0) \quad i i(L \quad 1, t) \quad i i(s, 0)
$$

By substituting (12) and (15) into (3), the probabilities of $P_{0}$ and $P_{1}$ are calculated by:

$P_{0}(s, t)=\frac{1}{M N} i i_{N}(s, t)$

$P_{1}(s, t)=\frac{1}{M N} F(s+1, t+1)$

By substituting (1) and (3) into (4), the average vector of 0 is calculated by:

$$
\begin{aligned}
& { }_{0}(s, t)=\left(\begin{array}{ll}
0,0 & 0,1
\end{array}\right)^{\mathrm{T}}=\square s{ }_{\square}^{\square} \square_{i=0} \square_{j=0} \frac{i p_{i j}}{P_{0}}, \square_{i=0}^{s} \square_{j=0}^{t} \frac{j p_{i j} \square^{\mathrm{T}}}{P_{0}} \square \\
& \begin{aligned}
& \square \\
&= \square s \square_{i=0} \square_{j=0} \frac{i c_{i j}}{\square_{i=0}^{t} \square_{j=0} c_{i j}}, \square_{i=0} \square_{j=0}^{t} \frac{j c_{i j}}{\square_{i=0}^{t}} \square_{j=0} c_{i j} \square \\
& \square
\end{aligned}
\end{aligned}
$$

By substituting (12) and (13) into (17), we calculate the average vector of ${ }_{0}$ by:

Average vector of target:

${ }_{0}(s, t)=\frac{\square i i_{O}(s, t)}{\square i i_{N}(s, t)}, \frac{i i_{G}(s, t)}{i i_{N}(s, t)} \square^{\mathrm{T}}$

Similarly, the average vector of $\quad$ is calculated using (19). Average vector of background:

${ }_{1}(s, t)=\left({ }_{1,0}, \quad{ }_{1,1}\right)^{\mathrm{T}}=\frac{\square F_{O}(s+1, t+1)}{\square F_{N}(s+1, t+1)}, \frac{F_{G}(s+1, t+1)}{F_{N}(s+1, t+1)} \square$

The total average vector of 2D histogram:

$T_{T}=\left(T_{T, 0}, \quad T_{, 1}\right)^{\mathrm{T}}=\frac{\square i i_{O}(s+1, t+1)}{M \square N}, \frac{i i_{G}(s+1, t+1)}{M \square N} \square^{\mathrm{T}}$ 
After the average vector is calculated using (18), (19) and (20), the trace of $\mathrm{SB}(\mathrm{s}, \mathrm{t})$ can be calculated using (7). The optimal threshold value $\left(s^{*}, t^{*}\right)$ can be determined by traversing the solution space only once, which reduces the computational complexity and the number of dimensions of the solution space.

\section{RESULTS AND DISCUSSION}

Windows Core i7 processor, $16 \mathrm{~GB}$ memory, $2.20 \mathrm{~Hz}$ flash memory and $1 \mathrm{~TB}$ hard disk are used as the hardware configuration of the experiment, PASCAL VOC2012 is used as the database image source, and 500 images are selected as the training set on the Simulink simulation platform to train the design platform. The maximum number of iterations is 5000 , the initial learning rate is 0.1 , and the learning rate change factor is 0.2 . After training, 400 images are randomly selected as the test. The simulation experiments were performed to compare the segmentation results and running time of the proposed algorithm in this work with those of two algorithms adopted in other studies [3] [4]. Here, we chose two images, including the image of a girl of size $256 \times 256$ pixels and the noisy image of E.coli cells of size $178 \times 178$ pixels and the image of the weld of size $256 \times 256$ pixels. The gray level gradient of the neighborhood is taken over a $3 \times 3$ window. The segmentation results are shown in Fig. 4, Fig. 5 and Fig. 6. Among them, the image segmentation in Fig. 6 is more important. From the experimental figure, it can be found that the method in literature [3] is interfered by more background illumination, which leads to more spots identified, and the effect of target segmentation is not good; The method in literature [4] can clearly segment the target, but the edge after segmentation is not clear enough, and there are more fuzzy edges; However, this method avoids the interference of uneven illumination, realizes more accurate target recognition and segmentation, and the segmentation edge is clearer, which has better application performance.

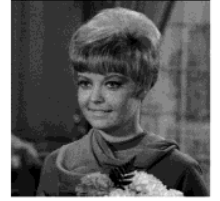

(a) Original image

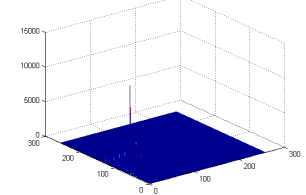

(b)2D histogram

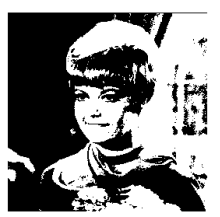

(c) Result of using the algorithm in [3]

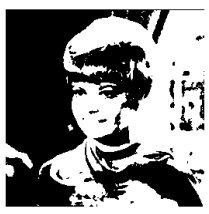

(d) Result of using the algorithm in [4]

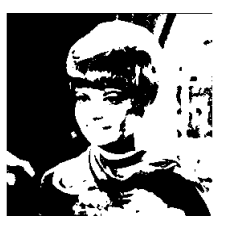

(e) Result of using the proposed algorithm
Fig. 4 segmentation results of the image of a girl
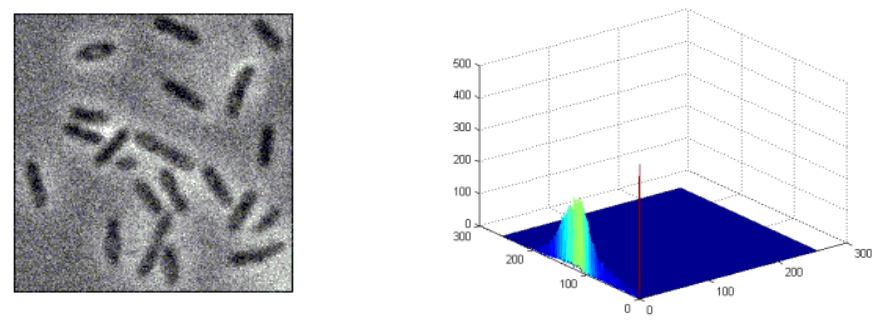

(a) Original image

(b) 2D histogram
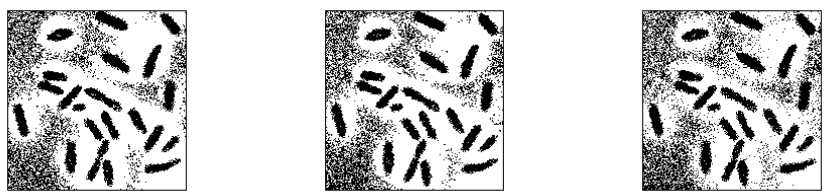

(c) Algorithm in [3]

(d) Algorithm used in [4]

(e) Proposed algorithm

Fig. 5 segmentation results of the noisy image of E.coli cells

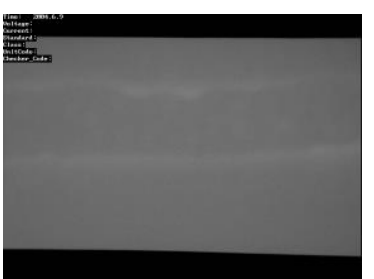

(a) Original image

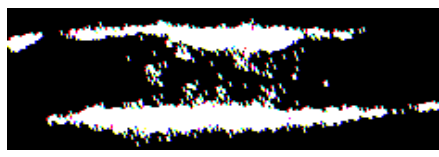

(c) Algorithm in [3]

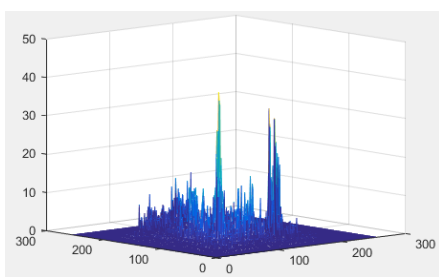

(b) 2D histogram

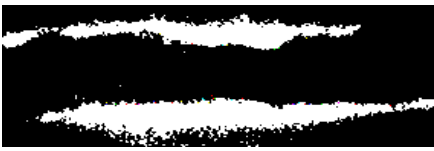

(d) Algorithm used in [4]

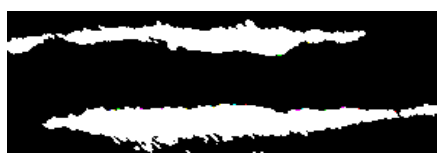

(e) Proposed algorithm

Fig. 6 segmentation results of the image of the weld

The optimal threshold values determined by three algorithms are listed in Table I. As shown in Figs. 4-6, the algorithms used in other studies had better segmentation effects on large objects [3] [4], but they incorrectly recognized the parts in the background, which showed large variation in gray level, as small objects. Compared with the algorithms used in other studies [3] [4], the improved 2D Otsu method proposed in this work could recognize the objects which have relatively clear edges, high anti-noise effects, and better homogeneity in the interior of the region. As for the efficiency of the algorithm, the improved 2D Otsu method reduced the running time of the algorithm for segmenting an image, as shown in Table II, thereby satisfying the requirement for real-time image processing. 
Table. I Optimal threshold values determined by different algorithms

\begin{tabular}{|c|c|c|c|}
\hline & $\begin{array}{c}\text { Image of a } \\
\text { girl }\end{array}$ & $\begin{array}{c}\text { Noisy image of } \\
\text { E.coli cells }\end{array}$ & $\begin{array}{c}\text { Image of the } \\
\text { weld }\end{array}$ \\
\hline $\begin{array}{c}\text { Algorithm } \\
\text { in [3] }\end{array}$ & $(74,78)$ & $(115,134)$ & $(121,137)$ \\
\hline $\begin{array}{c}\text { Algorithm } \\
\text { in [4] }\end{array}$ & $(74,78)$ & $(116,133)$ & $(122,138)$ \\
\hline $\begin{array}{c}\text { Proposed } \\
\text { algorithm }\end{array}$ & $(74,87)$ & $(116,172)$ & $(122,164)$ \\
\hline
\end{tabular}

Table. II Running time (s) of different algorithms

\begin{tabular}{|c|c|c|c|}
\hline & $\begin{array}{c}\text { Image of a } \\
\text { girl }\end{array}$ & $\begin{array}{c}\text { Noisy image of } \\
\text { E.coli cells }\end{array}$ & $\begin{array}{c}\text { Image of } \\
\text { the weld }\end{array}$ \\
\hline $\begin{array}{c}\text { Algorithm in } \\
\text { [3] }\end{array}$ & 67.539 & 60.176 & 61.247 \\
\hline $\begin{array}{c}\text { Algorithm in } \\
\text { [4] }\end{array}$ & 2.551 & 2.533 & 2.522 \\
\hline $\begin{array}{c}\text { Proposed } \\
\text { algorithm }\end{array}$ & 0.072 & 0.072 & 0.072 \\
\hline
\end{tabular}

From the above experimental results, it can be seen that the proposed method can effectively segment images, and the optimal threshold for segmentation can be determined for different types of images. However, through the image segmentation of large objects, it is found that this method can correctly identify the background in the image, and the edge of image recognition is clear, and the performance of anti-noise interference is better. Most importantly, the method designed in this paper effectively reduces the running time of image segmentation.

\section{CONCLUSION}

The integral image is an intermediate representation for the fast calculation of the sum of gray levels or the average gray levels of an image, which has a great advantage of calculating gray levels in a given region of the image. Based on the gray level-gradient 2D histogram, we proposed an integral image-based fast 2D Otsu method. This new method utilizes the integral image to reduce the computational complexity of searching the optimal threshold, the running time and the amount of computation of the algorithm, and it is proved to be a fast, efficient, and real-time thresholding method for image segmentation.

\section{References}

[1] C. G. Rafael, "Digital image processing second edition," Beijing: Publishing House of Electronics Industry, 2007, pp. 8.

[2] N. Otsu, "A thresholding selection method from gray-level histograms," IEEE Trans System Man Cybernetic, vol. 9, pp. 62-66, 1979.

[3] J. Z. Liu and W. Q. Li, "The automatic thresholding of gray-level pictures via two-dimensional Otsu method," Acta Automatica Sinica, vol. 19, no. 1, pp. 101-105, 1993.
[4] Y. M. Hao and F. Zhu, "Fast algorithm for two-dimensional Otsu adaptive threshold algorithm," Journal of Image and Graphics, vol. 10, no. 4, pp. 484-488, 2005.

[5] J. L. Fan and F. Zhao, "Two-dimensional Otsu's curve thresholding segmentation method for gray-level images," Acta Electronic Sinica, vol. 35, no. 4, pp. 751-755, 2007.

[6] R. Mamdouh, H. M. El-Bakry, A. Riad, and N. El-Khamisy, "Converting 2D-medical image files "DICOM" into 3D- models, based on image processing, and analysing their results with python programming," WSEAS Transactions on Computers, vol. 19, pp. 10-20, 2020.

[7] R. Kountchev and R. Kountcheva, "Image Segmentation based on adaptive mode quantization and 2D histograms analysis," WSEAS Transactions on Signal Processing, vol. 15, pp. 121-128, 2019.

[8] S. A. Stankevich, M. A. Popov, S. V. Shklyar, K. Y. Sukhanov, and B. Sun, "Subpixel-shifted satellite images superresolution: software implementation," WSEAS Transactions on Computers, vol. 19, pp. 31-37, 2020.

[9] M. Li, H. X. Yang, J. B. Zhang, T. T. Zhou, and Z. H. Tan, "Image thresholding segmentation research based on an improved region division of two-dimensional histogram," Journal of Optoelectronics Laser, vol. 24, no. 7, pp. 1426-1433, 2013.

[10]Z. Y. He, L. N. Sun, and L. G. Chen, "Fast computation of threshold based on Otsu criterion," Acta Electronic Sinica, vol. 41, no. 2, pp. 267-272, 2013.

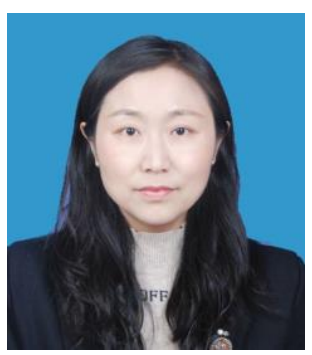

Yanli Tan, female, born in November 1978, associate professor, graduate student, signal and information processing of Zhongbei University. Department of electronic engineering of Taiyuan Institute of technology, image processing, has published 13 academic articles and participated in two scientific research projects.

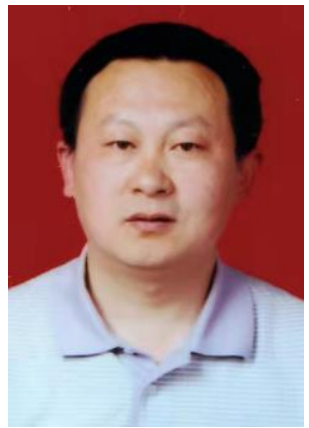

Yongqiang Zhao, male, born in October 1968, Professor, 
master, radio major of Taiyuan University of technology, science and Technology Department of Taiyuan Institute of technology, research direction: communication and information system, published more than 20 academic articles, participated in 5 scientific research projects and more than 10 teaching reform projects.

\section{Contribution description}

Yanli Tan proposed a fast two-dimensional Otsu method based on integral image based on gray gradient two-dimensional histogram.

Yongqiang Zhao simulation results show that the algorithm not only improves the calculation speed and real-time performance, but also has good image segmentation performance.

\section{Creative Commons Attribution License 4.0 (Attribution 4.0 International, CC BY 4.0)}

This article is published under the terms of the Creative Commons Attribution License 4.0

https://creativecommons.org/licenses/by/4.0/deed.en_US 